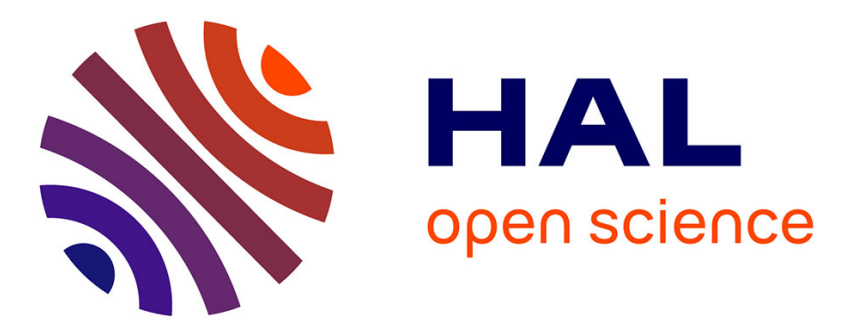

\title{
Determination of strain distribution and temperature gradient profiles from phase measurements of embedded fibre Bragg gratings
}

Xavier Chapeleau, Pascal Casari, Dominique Leduc, Y. Scudeller, Cyril Lupi, R. Le Ny, C. Boisrobert

\section{To cite this version:}

Xavier Chapeleau, Pascal Casari, Dominique Leduc, Y. Scudeller, Cyril Lupi, et al.. Determination of strain distribution and temperature gradient profiles from phase measurements of embedded fibre Bragg gratings. Journal of Optics A: Pure and Applied Optics, 2006, 8, pp.1-7. 10.1088/14644258/8/9/011. hal-01006728

\section{HAL Id: hal-01006728 \\ https://hal.science/hal-01006728}

Submitted on 27 Jan 2018

HAL is a multi-disciplinary open access archive for the deposit and dissemination of scientific research documents, whether they are published or not. The documents may come from teaching and research institutions in France or abroad, or from public or private research centers.
L'archive ouverte pluridisciplinaire HAL, est destinée au dépôt et à la diffusion de documents scientifiques de niveau recherche, publiés ou non, émanant des établissements d'enseignement et de recherche français ou étrangers, des laboratoires publics ou privés. 


\title{
Determination of strain distribution and temperature gradient profiles from phase measurements of embedded fibre Bragg gratings
}

\author{
X Chapeleau ${ }^{1}$, P Casari $^{2}$, D Leduc $^{1}$, Y Scudeller $^{3}$, C Lupi $^{1}$, \\ $\mathrm{R}$ Le $\mathrm{Ny}^{1}$ and $\mathrm{C}$ Boisrobert ${ }^{1}$ \\ ${ }^{1}$ Institut de Recherche en Electronique et Electrotechnique de Nantes Atlantique, Université \\ de Nantes, 2 rue de la Houssinière, 44300 Nantes, France \\ ${ }^{2}$ Institut de Recherche en Génie Civil et Mécanique UMR CNRS 6183, Université de Nantes, \\ 2 rue de la Houssinière, 44300 Nantes, France \\ ${ }^{3}$ Laboratoire de Génie des Matériaux et Procédés Associés, Ecole Polytechnique de \\ l'Université de Nantes, rue Christian Pauc, 44306 Nantes, France
}

\begin{abstract}
In this paper, a powerful method for measuring non-uniform strain is reviewed and extended to the measurement of temperature gradients inside an epoxy part. The sensor is an embedded fibre Bragg grating and the method is based on the measurements of the grating phase obtained by combining optical low coherence interferometry and an inverse scattering algorithm. This method has a great potential and for the first time experimental results obtained for both strain distribution and temperature gradient are presented. By applying a tensile test, strain distribution measurements are first compared with finite element simulations to validate previous published results. Second, original temperature gradient measurements in a solid body are shown. The results are in good accordance with reference measurements achieved with thermocouples incorporated into the body.
\end{abstract}

Keywords: fibre Bragg grating sensors, interferometry

\section{Introduction}

Since the 1990s, research in sensors based on optical fibre Bragg gratings (FBGs) has developed new sensing systems [1-3]. The main advantages of this technology are small size, light weight, high sensitivity, easily multiplexability, immunity to electromagnetic perturbation, and resistance to corrosion and fatigue. Systems based on FBG sensors have already been installed and are commercially available in large scale practical applications [4] such as bridges, mines, marine vessels, vehicles, aircraft, ....

An FBG is a segment of single-mode or multimode fibre in which a periodic modulation of the core refractive index is written by exposure to a spatial pattern of ultraviolet light.
According to Bragg's law, when an FBG is illuminated by a broadband source, only one wavelength (called the Bragg wavelength, $\left.\lambda_{B}\right)$ is preferentially reflected:

$$
\lambda_{\mathrm{B}}=2 n_{\mathrm{co}} \Lambda
$$

where $n_{\mathrm{co}}$ is the effective index of the fundamental mode and $\Lambda$ the period of the index modulation. The sensing principle is related to the optical properties of the FBG. When an FBG is subjected to strain and/or temperature changes, $n_{\mathrm{co}}$ and $\Lambda$ are modified and so the Bragg wavelength is shifted. Thus, for a temperature change of $\Delta T$, the corresponding Bragg wavelength shift is given by

$$
\Delta \lambda_{\mathrm{B}_{T}}=\lambda_{\mathrm{B}}(\alpha+\xi) \Delta T
$$


where $\alpha$ is the coefficient of thermal expansion and $\xi$ is the fibre thermo-optic coefficient. For an applied longitudinal strain $\epsilon$, the Bragg wavelength shift is given by

$$
\Delta \lambda_{\mathrm{B}_{\epsilon}}=\lambda_{\mathrm{B}}\left\{1-\frac{n_{\mathrm{co}}^{2}}{2}\left[P_{12}-v\left(P_{11}+P_{12}\right)\right]\right\} \epsilon
$$

where $P_{i j}$ are the components of the fibre-optic strain tensor and $v$ is Poisson's ratio. According to (2) and (3), strain or temperature changes can then be deduced from the Bragg wavelength shift measurement.

To achieve good sensor performances, it is crucial to determine with high precision the wavelength shift. A wavelength resolution of about $1 \mathrm{pm}$ is required to resolve a temperature change of $0.1^{\circ} \mathrm{C}$ or a strain change of $1 \mu \epsilon$. Investigation on high resolution interrogation methods led to the development of a certain number of techniques. The most common are based on the use of either an edge filter or a tuneable filter [5]. An interferometric scanning method [6] has also been reported and its high dynamic resolution demonstrated. In spite of the high precision of these wavelength shift detection techniques, they can only be used if the strain is uniform or if there is no temperature gradient along the grating. In the opposite case, the narrow band spectrum of the reflection coefficient becomes relatively broader and comprises multiple peaks. The FBG spectral shape is then more complicated to interpret so that it cannot be directly related to the strain or the temperature gradient along the grating. This problem becomes crucial for the so-called 'fibre-optic smart structures' [7] where FBGs are embedded into a complex structure to monitor its strain or temperature distribution and to check the structural integrity. This is particularly obvious for long gratings embedded into complex structures such as textile-reinforced composites in which the manufacturing process may induce non-uniform micro-mechanical deformation.

Several studies have focused on the determination of nonuniform strain along an FBG. Two first approaches based on the reflection spectrum [8] and the phase spectrum [9] of the FBG were proposed near the middle of the 1990s. However, they are valid only when the strain profile is monotonic and the sign of its slope is known. To overcome these difficulties, a method [10] based on the Fourier transform of the complex reflection coefficient of FBG was proposed in 1998. However, this technique also presents two drawbacks: the reflectivity level cannot be higher than $30 \%$ and the method is very sensitive to system noise. In 2000 , a time-frequency signalanalysis technique [11] demonstrated its efficiency, and it is free from the limitations mentioned above and can be applied without any restrictions on the strain profile. Nevertheless, this method does not work properly for FBGs in which different zones along the length of the grating couple with the same local Bragg frequency when the coupling strength is too strong. In 2002, a new approach based on a genetic algorithm was developed [12]. This method gives good results but its performance depends on the number of unknown parameters and on the choice of their range. Since this algorithm executes a multidirectional search that simultaneously analyses many possible solutions, the computation time is generally very long. In 2005, a new method which allows a direct reconstruction of the strain without any assumptions and a computational

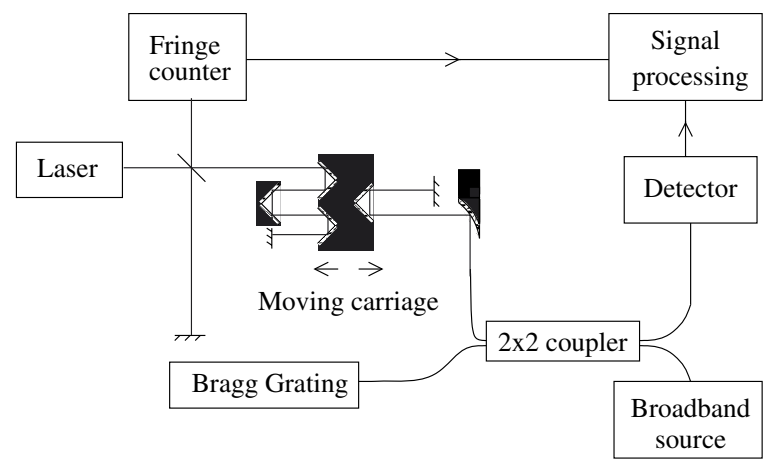

Figure 1. Optical low-coherence interferometer.

time of only of a few seconds was proposed [13]. It is based on the measurement of the complex impulse response of FBG by optical low-coherence interferometry (LCI) and the retrieval of the local Bragg wavelength by the layer-peeling (LP) technique. Axial strain distribution measurements using uniform FBG sensors can be measured accurately using this method. However, it was never applied to temperature gradient profile measurement.

In this paper, we propose to show the great potential of this method while presenting both experimental results obtained for strain distribution and temperature gradient using uniform or chirped gratings. Indeed, we extend the method for the first time to temperature distribution measurements and demonstrate its efficiency. For this, we have developed a slightly different method, which is based on the measurement of the complex reflection coefficient of FBG by LCI and the retrieval of the phase of the grating by LP. In section 2, we carefully describe our method and then we will show how a non-uniform strain distribution or temperature gradient can be extracted from the phase of the grating. In sections 3 and 4, we will present and discuss the experimental results obtained respectively for a non-uniform strain distribution and a temperature gradient in a steady state condition.

\section{Method for strain or temperature distribution measurements}

\subsection{Description of the LCI technique}

The interferometer is a Michelson-type interferometer assembled around a 'two-by-two' single-mode fibre coupler (figure 1). The test arm is connected to the grating sample to be tested. The wave, which propagates along the reference arm, is bent on a sliding corner cube reflector and oriented toward a fixed plane mirror. The He-Ne laser fringe counting system output is used to sample the infrared interferogram every $80 \mathrm{~nm}$ $\left(\lambda_{\mathrm{He}-\mathrm{Ne}} / 8\right)$.

The signal received by the detector is the superposition of the DC signal corresponding to the intensities reflected by each arm and the AC signal created by the interferences of the two reflected waves. The receiver is AC-coupled and amplifies only the oscillations. Theoretically, the variable part of the intensity is

$$
I(x)=\int_{-\infty}^{+\infty} S(\sigma) r(\sigma) \exp (-8 \mathrm{i} \pi \sigma x) \mathrm{d} \sigma
$$


where $x$ is the displacement of the moving carriage, $\sigma$ the wavenumber, $S(\sigma)$ the source spectral power density and $r(\sigma)$ the complex amplitude reflection coefficient.

Relation (4) shows that $r(\sigma)$ can easily be computed from the Fourier transform of the interferogram when the power spectral density of the source is known. From the square of the modulus of $r(\sigma)$ the spectrum of the FBG is obtained, and from the phase of $r(\sigma)$ the group delay and the chromatic dispersion of the FBG can be calculated. This reflectometric method has already been thoroughly tested and reported $[14,15]$. We have demonstrated the repeatability of fibre Bragg grating reflectance and group delay LCI measurements and showed their accuracy by comparison with the results of the NIST Telecom Round Robin. Accuracy in these measurements is absolutely required to determine the index profile modulation and the phase of FBG using the layer-peeling method.

\subsection{Description of layer-peeling algorithm}

The layer-peeling inverse method has been reported in many recent publications [16-18]. It is a powerful tool for the design of the index profile from a theoretical complex reflection coefficient. Indeed, the LP algorithm takes into account multiple reflections into the FBG so that it can reconstruct correctly the index profile of gratings whose reflection coefficient is high but lower than $90 \%$. We point out the fact that we could also use other inverse scattering algorithms [19] based on integral formulations which give similar results but they are greedy in computational time.

The LP algorithm is based on the assumption that the index change (caused by UV exposure) is assumed to be quasisinusoidal:

$$
\begin{aligned}
n(z) & =n_{\mathrm{co}}+\Delta n_{\mathrm{DC}}(z)+\Delta n_{\mathrm{AC}}(z) \\
& \times \cos \left[\frac{2 \pi}{\Lambda} z+\frac{2 \pi}{\Lambda^{2}} \int_{0}^{z}\left(\Lambda\left(z^{\prime}\right)-\Lambda\right) \mathrm{d} z^{\prime}\right]
\end{aligned}
$$

where $\Delta n_{\mathrm{AC}}$ is the index modulation amplitude, $\Delta n_{\mathrm{DC}}$ the variation of the refractive index averaged over a single period of the grating, $n_{\mathrm{co}}$ the refractive index of the core of the fibre and $\Lambda(z)$ is the grating's period. Then, FBG is discretized with $N$ layers, so that the index change can be considered as constant. We also assume that the fibre is lossless and guides one single mode.

The quantity obtained with the LP algorithm is the complex coupling coefficient $q(z)$. The modulus of $q(z)$ is proportional to the index modulation amplitude $\Delta n_{\mathrm{AC}}$ and the phase of $q(z)$ is linked to the variation of the refractive index $\Delta n_{\mathrm{DC}}$ and to the variation of the grating's period:

$\Psi(z)=-\frac{2 \pi}{n_{\mathrm{co}} \Lambda} \int_{0}^{z} \eta \Delta n_{\mathrm{DC}}\left(z^{\prime}\right) \mathrm{d} z^{\prime}-\frac{2 \pi}{\Lambda^{2}} \int_{0}^{z}\left(\Lambda\left(z^{\prime}\right)-\Lambda\right) \mathrm{d} z^{\prime}$

where $\eta$ is the overlap factor of the fibre. In this paper, we call it the phase of the grating.

With the combination of our LCI technique and LP algorithm the phase of the grating is measured with a spatial resolution of $20 \mu \mathrm{m}$, and from this quantity the strain distribution or the temperature gradient along the grating can be deduced as shown in the following section.

\subsection{Description of strain or temperature distribution measurements}

Let us call $\Psi_{0}(z)$ the phase of the free grating. If an external constraint, either a temperature gradient $\Delta T(z)$ or an axial tension which induces a strain $\epsilon(\mathrm{z})$ supposed as non-uniform, is applied to the FBG, then both the period of the grating and the core refractive index are affected. In the linear limit, the variations of the grating's period are related to $\Delta T(z)$ or to $\epsilon(\mathrm{z})$ by

$$
\begin{gathered}
\frac{\delta \Lambda(z)}{\Lambda}=\alpha \Delta T(z) \\
\frac{\delta \Lambda(z)}{\Lambda}=\epsilon(z) .
\end{gathered}
$$

By the photo-elastic effect or photo-thermal effect, the variations of the refractive core index are

$$
\begin{gathered}
\delta n_{\mathrm{DC}}(z)=n_{\mathrm{co}} \xi \Delta T(z) \\
\delta n_{\mathrm{DC}}(z)=-\frac{n_{\mathrm{co}}^{3}}{2}\left[P_{12}-v\left(P_{11}+P_{12}\right)\right] \epsilon(z) .
\end{gathered}
$$

Taking into account these two effects, the temperature gradient or the variation of the strain along the FBG can be retrieved from the measurement of the phase of the grating with and without external constraints:

$$
\begin{aligned}
\Delta T(z) & =K_{\Delta T} \frac{\mathrm{d}\left[\Psi(z)-\Psi_{0}(z)\right]}{\mathrm{d} z} \\
\epsilon(z) & =K_{\epsilon} \frac{\mathrm{d}\left[\Psi(z)-\Psi_{0}(z)\right]}{\mathrm{d} z}
\end{aligned}
$$

where $K_{\Delta T}$ is a constant which depends on the thermo-optic and thermal expansion coefficients of the FBG and $K_{\epsilon}$ is a constant which depends on the photoelastic coefficients and Poisson's ratio of the FBG

To summarize, the method is based on the measurements of the phase of the grating. These quantities are obtained using the combination of the LCI technique and the LP algorithm previously discussed in sections 2.1 and 2.2. To retrieve the strain or the temperature gradients along the grating, the scheme of the method is as follows.

(i) The phase $\Psi_{0}(z)$ of the grating without strain distribution or temperature gradient is determined.

(ii) The phase $\Psi(z)$ of the grating with strain distribution or temperature gradient is measured.

(iii) The strain distribution or the temperature gradient are obtained by using respectively the formulae (9) and (10).

This last step requires us to calculate a discrete derivative which is not straightforward due to the noise in the experimental data. To reduce it, we choose to take the average of ten measurements of the phase. Nevertheless, the result of the derivative still shows some ripples which have no significant physical meaning. So, the results of strain or the gradient of temperature measurements presented in this paper are obtained by applying a smoothing filter based on the least squares.

The method presented previously cannot be applied if there are both an unknown strain distribution and an unknown temperature gradient. Indeed, these two effects are added, so that it is impossible to distinguish them. To validate our method we then studied them separately, and in the next section results of strain distribution measurements will be first shown 


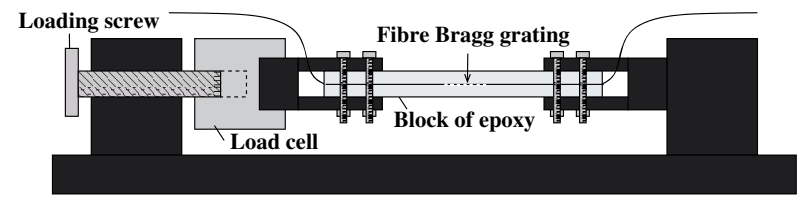

Figure 2. Tensile test set-up.

\section{Strain distribution measurement}

\subsection{The experimental procedure and the finite element simulations}

3.1.1. The experimental procedure and calibration. To test the method of strain distribution measurement proposed in this paper, an FBG without fibre coating was embedded in a rectangular block of epoxy with the following dimensions: $200 \times 32 \times 8 \mathrm{~mm}^{3}$. The FBG is $11 \mathrm{~mm}$ long and is located in the middle of the specimen and oriented in the direction of the length axis. It was fabricated in a SMF28 fibre by means of the phase mask technique and it is a uniform grating.

The epoxy resin is Axson Epolam 2020, which cures at room temperature. The choice of this resin is because of a good compatibility with the FBG regarding the bonding and the easiness of insertion. During filling and polymerization, the FBG is maintained under slight tension inside the mould in order to ensure its location in the middle of the sample. The fittings at the ends of the sample allow the fibre to emerge straight.

After removal from the mould, the FBG sensor was calibrated. Indeed, it is necessary to determine the coefficient $K_{\epsilon}$ (10) which is characteristic of the grating used. For this, a preliminary tensile test was performed. The tensile test set-up (see figure 2) is an aluminium frame with a ball joint on each side, a load cell placed at one end for the load measurement and a loading screw able to apply the load. The FBG is then stretched with the sample. This small device was easily placed along the interferometer during measurements. By determining the phase of the FBG for different strains measured with an extensometer placed on the sample, a coefficient $K_{\epsilon}$ equal to about $1.1 \times 10^{2} \mu \epsilon \mathrm{mm} \mathrm{rad}^{-1}$ was found. After this calibration, the sample was drilled with one hole on each side of the FBG (see figure 3), which induces a symmetrical strain gradient in the area of the FBG sensor. Then, new tensile tests were performed.

3.1.2. The finite element simulation. A numerical mechanical model has been implemented with the finite element method in the code CAST3M [20]. It consists in representing the epoxy sample as a plate under the hypothesis of plane stresses. The FBG is not included in the model because of its small diameter and its negligible contribution to the stiffness of the sample. The geometry is the one of the sample but two symmetries are used in order to simplify the problem. One quarter of the sample is represented in figure 3.

\subsection{Results and discussions}

Figure 4 shows the amplitude reflection coefficient of the FBG before and after it was embedded. The polymerization of

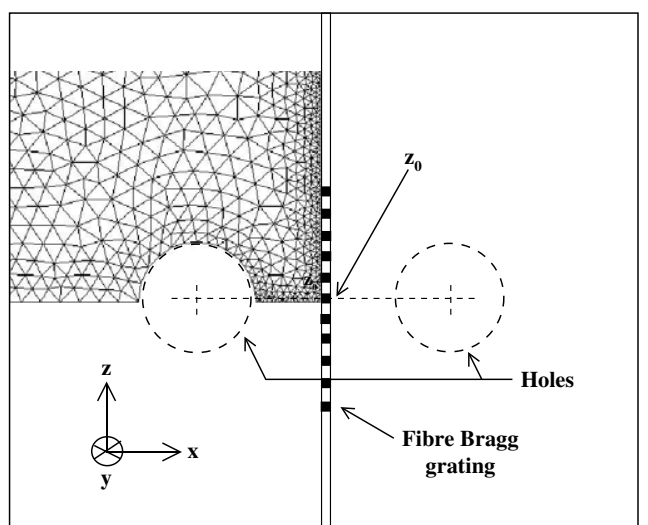

Figure 3. Scheme of the sample and quarter of the finite element mesh.

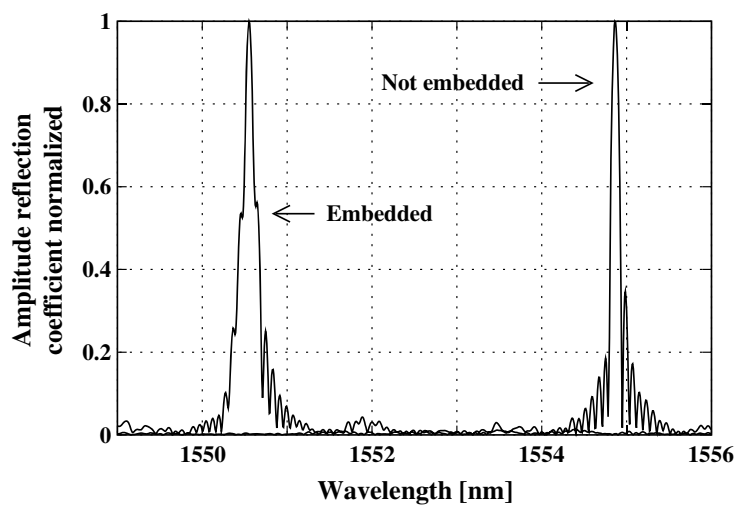

Figure 4. Amplitude reflection coefficient of FBG before and after embedment.

the resin causes a shift of the Bragg wavelength of about $4.45 \mathrm{~nm}$ and the spectrum becomes broader. This widening can be explained by the shrinkage of the resin and the parttool interactions. They imply deformation of the FBG which changes its properties, thus causing a birefringent effect. This effect leads to a change in the refractive index so that two plane-polarized waves will propagate along the grating. The response of the FBG is then composed of two peaks. In our case, we cannot distinguish them in figure 4 , so the birefringent effect due to the embedment and the polymerization process is small and we can neglect it.

Figure 5(a) shows the amplitude reflection coefficient of the FBG loaded under different tensile loads. These measurements have been obtained by the optical low coherence interferometer described in section 2.1. The higher the force, the more the spectrum is shifted toward higher wavelengths and the more it is distorted. This distortion is obviously due to a non-uniform strain distribution along the grating.

Before measuring the strain distribution by using the method described in section 2.3, we verified that the birefringence induced by the axial load is insignificant. When the sample is loaded, the presence of the holes creates two transverse stresses $\sigma_{x x}$ and $\sigma_{y y}$, respectively perpendicular and parallel to the axes of the holes, which are not equal. As a consequence, the refractive index will change by the 


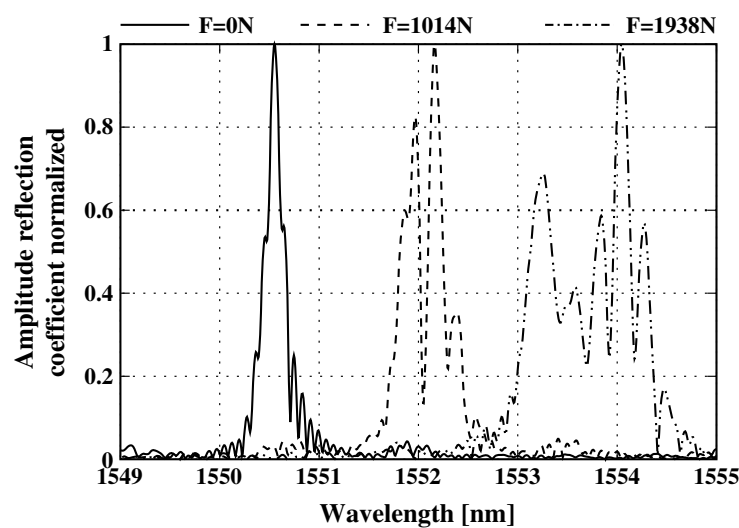

(a) Amplitude reflection coefficient

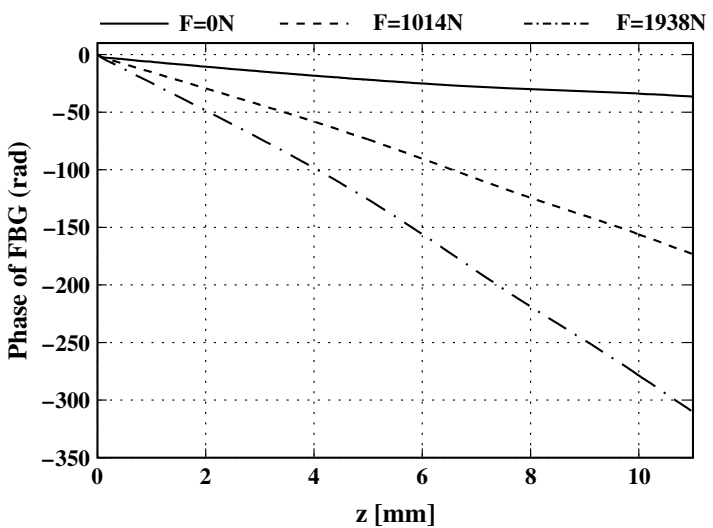

(b) Phase

Figure 5. Amplitude reflection coefficient and phase of FBG for several tensile loads.

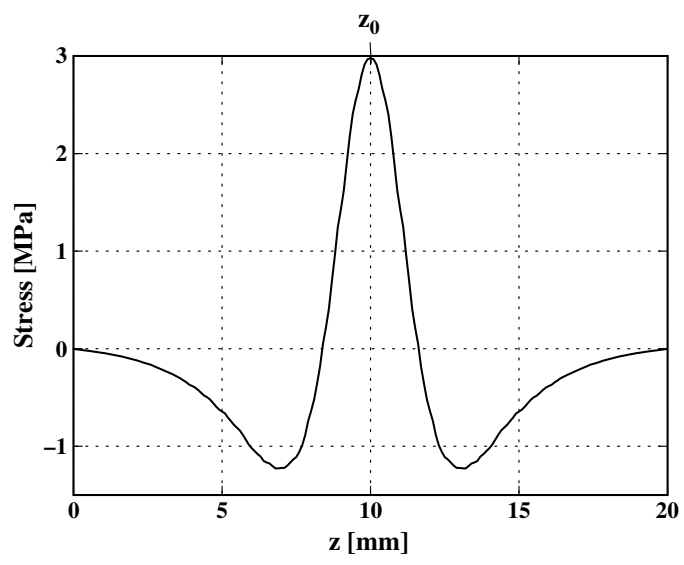

Figure 6. Transverse stress $\sigma_{x x}$ obtained by the finite element simulation.

photoelastic effect and become $\Delta n_{x}$ and $\Delta n_{y}$ for the $x$ - and $y$-polarization respectively. A numerical simulation by the finite element method was made to evaluate the transverse stress $\sigma_{x x}$ (figure 6) with a maximum of $3 \mathrm{MPa}$, while the $z z$ longitudinal stress average on the sample is set to $50 \mathrm{MPa}$. The transverse stress $\sigma_{y y}$ was set to zero through the plane stress assumption. This approach is widely accepted in the field of structural mechanics when the thickness of the structure is small compared to the width. Effective change in refractive index in both planes could be derived using photoelasticity theory [21]:

$$
\Delta n_{x}(z)-\Delta n_{y}(z)=\frac{n_{\mathrm{co}}^{3}}{2 E} K\left(\sigma_{x x}-\sigma_{y y}\right)
$$

where $E$ is Young's modulus for the fibre and $K$ is a constant which depends on the photoelastic coefficients and Poisson's coefficient. Using $K=0.1743, E=74 \mathrm{GPa}$ and $n_{\mathrm{co}}=1.45$, the value of the birefringence due to the load of the sample cannot exceed $1 \times 10^{-5}$, in the worst case. This value is sufficiently small that we can neglect the birefringence effect in strain distribution measurements.

From the complex reflection coefficients, the phase of the FBG is then determined by using the layer-peeling algorithm

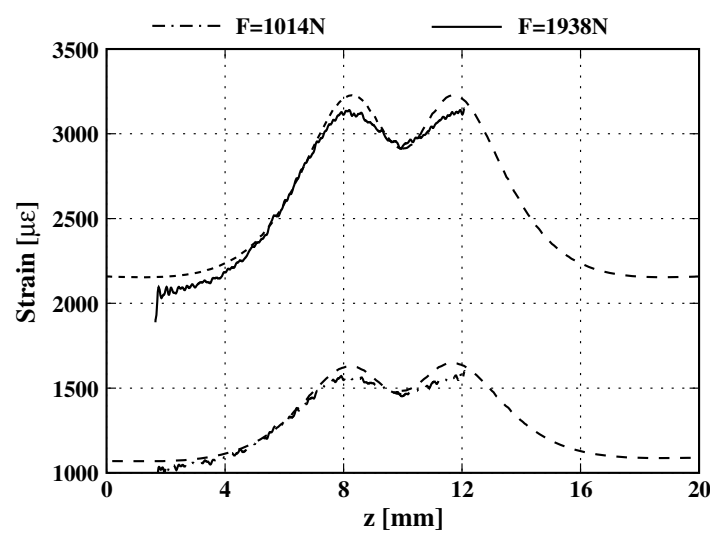

Figure 7. Strain distribution measurements from the phase grating (solid curves) and from the finite element simulations (dashed curves).

(see section 2.2). Figure 5(b) shows them for different tensile loads. The strain distribution was then calculated using (10) and the results are plotted in figure 7 . The strain curves are not monotonic and correspond to a non-uniform strain distribution as expected. With a difference below $5 \%$, a good agreement is observed between simulated (dashed curves) and experimental distributions.

\section{Temperature gradient measurement}

\subsection{Experimental procedure and calibration}

The experimental set-up shown in figure 8 has been designed in order to create a carefully controlled temperature gradient in a solid body where the FBG sensor is incorporated. The body is formed from polymethyl methacrylate-based organic material (PMMA).

As a preliminary, a cylindrical polymethyl methacrylate block (diameter of $80 \mathrm{~mm}$ and thickness of $20 \mathrm{~mm}$ ) was cut in half and a micro-groove with a cross-section of about $200 \mu \mathrm{m} \times 200 \mu \mathrm{m}$ was machined in the middle of one half. An FBG sensor was then placed in this micro-groove and the two half spherical blocks were bonded. A temperature gradient 


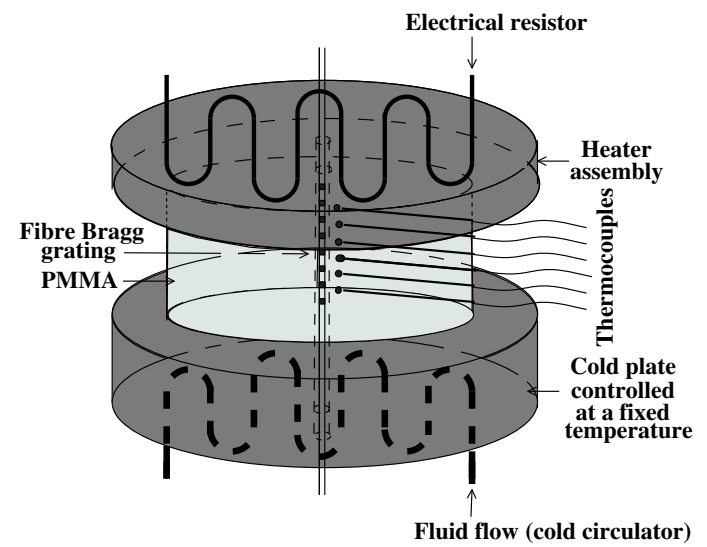

Figure 8. Experimental set-up of temperature gradient.

was created by placing the PMMA cylinder between a heater assembly and a brass cold plate controlled at fixed temperature by a circulator (see figure 8 ). The heater was composed of an electrical resistor brazed on a brass plate. In steady state conditions, the thermal gradient becomes uniform because of the high thermal conductivity of brass and the size of the cylinder.

To demonstrate that our method can be applied with any type of gratings, the FBG used in this temperature gradient experiment is a chirped fibre Bragg grating. It is $10 \mathrm{~mm}$ long and its chirp is equal to $1.5 \mathrm{~nm} \mathrm{~cm}^{-1}$. Before embedding the FBG sensor in the block of polymethyl methacrylate, it is necessary to calibrate it. The goal of this calibration is to determine the coefficient $K_{\Delta T}$ (9) which is characteristic of the FBG sensor used. The grating was embedded with a thermocouple in an isothermal brass block. In order to calibrate the FBG sensor, the block was carefully maintained at different temperatures by using a heater. The dimensions of the block are sufficient to ensure a uniform temperature around the grating and the reference thermocouple. By determining the phase of the FBG sensor for different temperatures measured with the thermocouple, we found a coefficient $K_{\Delta T}$ equal to $11.8^{\circ} \mathrm{C} \mathrm{mm} \mathrm{rad}{ }^{-1}$.

\subsection{Results and discussions}

In figure 9(a), the solid curve shows the spectrum of the FBG without any temperature gradient. In contrast to a uniform grating, a chirp grating has a broader band-width. The full width at half maximum (FWHM) is equal to $1.4 \mathrm{~nm}$. With the temperature gradient, the dashed curve shows that the spectrum is slightly shifted and the FWHM decreases by about $160 \mathrm{pm}$. These effects are due to the modifications of the chirp of the grating and of the refractive index distribution by the temperature gradient. These modifications depend on the direction of the grating period compared to the temperature gradient. In other words, if the grating had been turned in the other direction, the FWHM would have probably increased.

The solid curve of figure 9(b) shows the phase of the grating without the temperature gradient. With the temperature gradient (the dotted curve) the curve of the phase becomes less rounded and we can interpret it like a reduction of the chirp of the grating. It is in agreement with the decrease of band-width observed in figure 9(a).

From these phase measurements, the temperature gradient was calculated with the help of (9) and the result, the solid curve, is shown in figure 10 . The measured temperature gradient is linear with a slope of $2{ }^{\circ} \mathrm{C} \mathrm{mm}^{-1}$ with a spatial resolution of $20 \mu \mathrm{m}$. The thickness of the PMMA cylinder is $20 \mathrm{~mm}$ and the length of the FBG sensor is about $10 \mathrm{~mm}$, so the grating used is too short to measure the entire temperature gradient. We chose to place the FBG sensor close to the heated plate but it is very difficult to locate it accurately. In order to be able to compare the gradient measurement with reference measurement achieved with thermocouples (in figure 10, each dot corresponds to a thermocouple), the curve was shifted only on the $Z$-coordinate. The agreement between the two results is very good and it demonstrates the validity of the LCI-LP method.

\section{Conclusion}

In this paper, we have described a powerful method for measuring non-uniform strain and temperature gradient with fibre Bragg gratings. It is based on the measurement of the

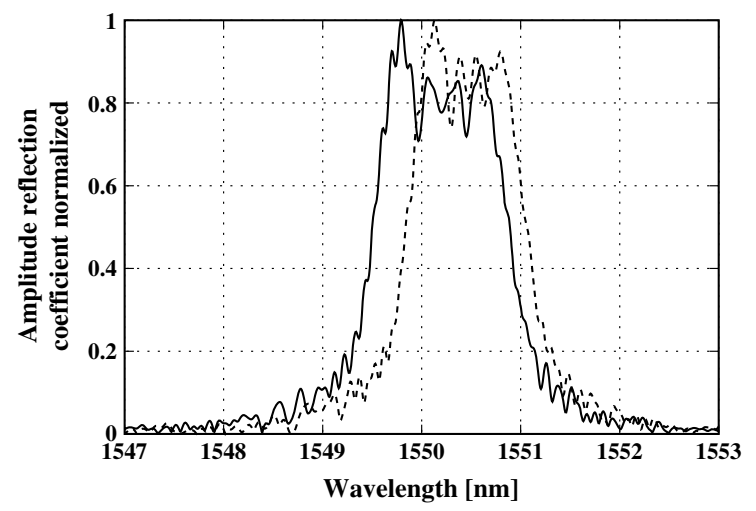

(a) Amplitude reflection coefficient

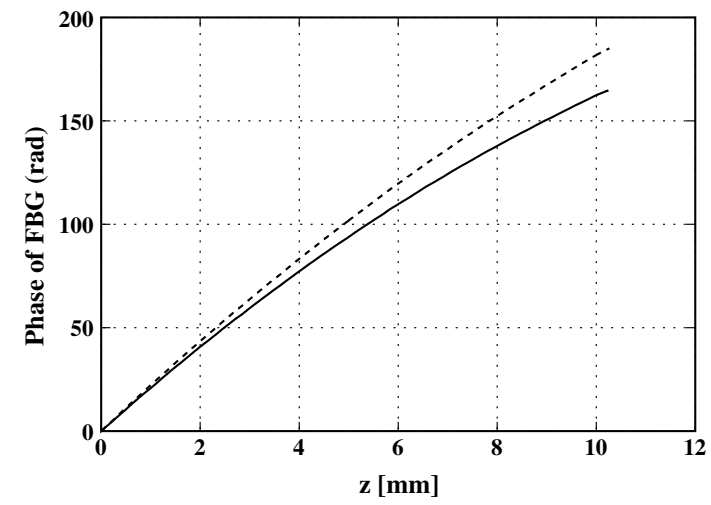

(b) Phase

Figure 9. Amplitude reflection coefficient and phase of FBG without (solid curves) and with (dotted curve) temperature gradient. 


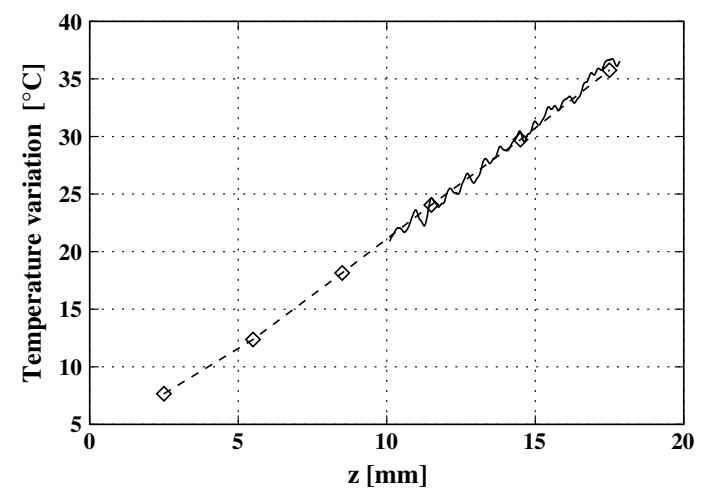

Figure 10. Measurement of the temperature gradient from the phase grating (solid curves) and from the thermocouples (dashed curve).

phase of the grating which can be obtained by combining the optical low coherence interferometry and the layer-peeling algorithm. Two measurements of the phase of the grating (with and without strain distribution or temperature gradient) are required and from the derivative of the difference of these phases, the strain or the gradient temperature along the grating can be retrieved.

By this method, we performed non-uniform strain distribution and temperature gradient measurements using uniform or chirped FBG sensors. The measured strain distribution was compared to finite element simulation and temperature gradient was compared with thermocouples. In each case, the agreement was excellent. This demonstrates the efficiency of the method.

So, it is a promising technique to quantify strain or temperature distributions inside composite parts. These measurements of gradients would lead to an accurate characterization of both the mechanical and thermal behaviours where classical techniques are only able to provide surface measurements.

\section{Acknowledgments}

We thank Patrick Derval and Michel Roche for their technical support.

\section{References}

[1] Rao Y-J 1997 In-fibre Bragg grating sensor Meas. Sci. Technol. 8 355-75

[2] Kersey A 1996 A review of recent developments in fibre optic sensor technology Opt. Fibre Technol. 2 291-317

[3] Kersey A, Davis M, Patrick H, LeBlanc M, Koo K, Askins C, Putnam M and Frieble E 1997 Fibre grating sensors J. Lightwave Technol. 15 1442-63
[4] Rao Y-J 1999 Recent progress in applications of in-fibre Bragg grating sensors Opt. Lasers Eng. 31 297-324

[5] Zhao Y and Liao Y 2004 Discrimination methods and demodulation techniques for fibre Bragg grating sensors Opt. Lasers Eng. 41 1-18

[6] Kersey A, Berkoff T and Morey W 1992 High-resolution fibre-grating based strain sensor with interferometric wavelength-shift detection Electron. Lett. 28 236-8

[7] Peters K, Studer M, Botsis J, Iocco A, Limberger H and Salathé R 2001 Embedded optical fibre Bragg grating sensor in a nonuniform strain field: measurements and simulations Exp. Mech. 41 19-28

[8] LeBlanc M, Huang S, Ohn M, Measures R, Guemes A and Othonos A 1996 Distributed strain measurement based on a fibre Bragg grating and its reflection spectrum analysis $O p t$. Lett. 21 1405-7

[9] Huang S, Ohn M and Measures R 1996 Phase-based Bragg intragrating distributed strain sensor Appl. Opt. 35 1135-42

[10] Huang S, Ohn M, LeBlanc M and Measures R 1998 Continuous arbitrary strain profile measurements with fibre Bragg gratings Smart Mater. Struct. 7 248-56

[11] Azaña J and Muriel M 2000 Reconstructing arbitrary strain distributions within fibre gratings by time-frequency signal analysis Opt. Lett. 25 698-700

[12] Casagrande F, Crespi P, Grassi A M, Lulli A, Kenny R P and Whelan M P 2002 From the reflected spectrum to the properties of a fibre Bragg grating: a genetic algorithm approach with application to distributed strain sensing Appl. Opt. 41 5238-44

[13] Giaccari P, Dunkel G, humbert L, Botsis J, Limberger H and Salathé R 2005 On a direct determination of non-uniform internal strain fields using fibre Bragg gratings Smart Mater. Struct. 14 127-36

[14] Chapeleau X, Leduc D, Lupi C, Le Ny R, Douay M, Niay P and Boisrobert C 2003 Experimental synthesis of fibre Bragg gratings using optical low coherence reflectometry Appl. Phys. Lett. 82 4227-9

[15] Leduc D, Chapeleau X, Lupi C, Le Ny R and Boisrobert C 2003 Accurate low-coherence interferometric relative group delay and reflectance measurements; characterization of a free space optics multiplexer/demultiplexer J. Opt. A: Pure Appl. Opt. 5 51-5

[16] Feced R, Zervas M and Muriel M 1999 An efficient inverse scattering algorithm for the design of nonuniform fibre Bragg gratings IEEE J. Quantum Electron. 35 1105-15

[17] Poladian L 2000 Simple grating synthesis algorithm Opt. Lett. 25 787-9

[18] Skaar J, Wang L and Erdogan T 2001 On the synthesis of fibre Bragg gratings by layer peeling IEEE J. Quantum Electron. 37 165-73

[19] Leduc D, Chapeleau X, Lupi C, Le Ny R and Boisrobert C 2005 Comparison of two inverse scattering algorithms for the experimental synthesis of fiber Bragg gratings Proc. 5th Int. Conf. on Inverse Problems in Engineering: Theory and Practice vol II (Leeds, UK: Leeds University Press) p L04

[20] http://www-cast3m.cea.fr

[21] Gafsi R and El-Sherif M 2000 Analyse of induced-birefringence effects on fibre Bragg gratings Opt. Fibre Technol. 6 299-323 